\title{
Control Moment Gyroscope (CMG) for Roll Stabilization of a Heavy Vehicle
}

Faruk Ünker ( $\square$ farukunker@gumushane.edu.tr)

Gümüşhane University https://orcid.org/0000-0002-9709-321X

\section{Research Article}

Keywords: Gyroscope, gyrostabilizer, control moment gyroscope, vehicle rollover, inverted pendulum.

Posted Date: November 8th, 2021

DOI: https://doi.org/10.21203/rs.3.rs-916038/v1

License: (9) This work is licensed under a Creative Commons Attribution 4.0 International License. Read Full License 


\title{
Control Moment Gyroscope (CMG) for Roll Stabilization of a Heavy Vehicle
}

\author{
Faruk ÜNKER \\ farukunker@gumushane.edu.tr \\ Department of Mechanical Engineering, Gümüşhane University ,29100 Gümüşhane, Turkey
}

\begin{abstract}
In this research, a moment propagated by a flywheel gyroscope is analysed as a control moment gyro $(\mathrm{CMG})$ to prevent vehicle rollover. When the torque of the rotating flywheel is controlled, specific sinusoidal motion occurs. It is observed that the sinusoidal gimbal motion causes to be a smallish vibration amplitude in the upright position of the sprung mass. Therefore, CMG maintains the rollover stability of the sprung mass with a steady motion. Besides, there is a simulation model using a CAE software (RecurDyn), which is built to validate the equations of motion.
\end{abstract}

Keywords: Gyroscope, gyrostabilizer, control moment gyroscope, vehicle rollover, inverted pendulum.
Nomenclature
g the gravitational acceleration
G the mass center of sprung mass
L the sprung's length of the mass centroid
$\mathrm{T}_{\text {sprung mass }}$ the kinetic energy of the sprung
$\mathrm{T}_{\text {gimbal_1,2 }}$ each gimbal's kinetic energy
$\mathrm{T}_{\text {flywheel_1,2 }}$ each flywheel's kinetic energy
$\mathrm{T}_{\text {total }} \quad$ vehicle's total kinetic energy
$\mathrm{V}_{\text {total }} \quad$ vehicle's total potential energy
$(\mathrm{X}, \mathrm{Y}, \mathrm{Z}) \quad$ the coordinates
$\theta \quad$ each gimbal's precession angle 


\begin{tabular}{|c|c|}
\hline$\theta_{0}$ & each gimbal's amplitude of precession angle \\
\hline$\varphi$ & the roll angle of sprung mass \\
\hline$\varphi_{0}$ & the sprung's amplitude of roll angle \\
\hline$\omega$ & the frequency of harmonic motion \\
\hline $\mathrm{m}_{\mathrm{b}}$ & the sprung mass \\
\hline $\mathrm{m}_{\mathrm{g}}$ & the gimbal mass \\
\hline $\mathrm{m}_{\mathrm{d}}$ & the flywheel mass \\
\hline $\mathrm{I}_{\mathrm{p}}$ & each flywheel's rotary inertia \\
\hline $\mathrm{I}_{\mathrm{o}}$ & each flywheel's moment inertia \\
\hline $\mathrm{I}_{\mathrm{gx}}, \mathrm{I}_{\mathrm{gy}}, \mathrm{I}_{\mathrm{gz}}$ & $\begin{array}{l}\text { the gimbal's principal inertia moments at the center of gimbal } \\
\text { mass }\end{array}$ \\
\hline $\mathrm{I}_{\mathrm{bx}}, \mathrm{I}_{\mathrm{by}}, \mathrm{I}_{\mathrm{bz}}$ & $\begin{array}{l}\text { the sprung's principal inertia moments at the center of the } \\
\text { sprung mass }\end{array}$ \\
\hline$\Omega$ & each flywheel's rotational velocity \\
\hline$\dot{\alpha}$ & the cornering speed \\
\hline $\mathrm{K}_{\varphi}$ & the stiffness coefficient of torsional spring \\
\hline $\mathrm{R}$ & the radius of the cornering of the vehicle \\
\hline $\mathrm{R}_{\mathrm{g}}$ & the radius of the cornering of each gyroscope \\
\hline
\end{tabular}

\section{Introduction}

Due to the high center of gravity, rollover is a serious safety issue for commercial or sport utility vehicles. In order to improve the stationarity condition of these vehicles, the complexity of active anti-rollover systems increases (Mirzaeinejad H and Mirzaei M, 2014; Takahashi J et al. 2013; Tchamna R et al., 2014). In recent years, many researchers have investigated safety control systems to prevent vehicle rollover by using electronic stability program (ESP), (Chen B. and Peng 
H, 2001; Ungoren AY and Peng H, 2004), longitudinal speed control (LSC), (Yoon J et al., 2009), an active suspension (Lee AY, 2002; Hac A, 2002). However, these control systems might not be adequate in mitigating rollover during cornering. The existing safety systems can indicate unwanted energy losses to reduce lateral acceleration by decreasing the longitudinal vehicle speed and fluctuating the purpose of the driver's operation with the additional yaw moment and yaw unstability.

The sprung mass of the vehicle should be investigated as an inverted pendulum problem. Various methods are used to stabilize a moving inverted pendulum in an upright position, such as by moving its body horizontally to balance its center of gravity (Irdayanti et al., 2020), using a control moment gyroscope (CMG) (Ünker, 2021) and applicating a reaction wheel (Larimi et al., 2015). The size of the force can cause the vehicle to fall while tilting its body to provide stability (Xu et al., 2016). Besides, the robust control algorithms improved for stabilization does not perfectly ensure a inverted pendulum-like vehicle although it consists of complex electromechanical systems. (Ruan and Chen, 2010; Lin et al., 2011). To cope with this restraint, a reaction wheel may be used to create a moment on the lateral axis. However, they are less stable and have less control moment with a constant angular velocity compared with CMG. In which, CMG can directly control the roll motion on the lateral axis of the sprung mass by providing a reactionless anti-roll torque as a rollover prevention energy reserve.

CMG can be used to balance the vehicle by tilting the gimbal of a gyroscope (Ünker, 2020; Ünker, 2021). CMG were used to generate continuous torque to 
avoid the vehicle from falling (Ünker, 2021). This controllable moment can be applied against constant (continuous) inertia forces (centrifugal force) to balance the sprung for a long time period. Besides, the high angle of the gimbal needs the low rotational velocity of flywheel.

In this paper, a innovator idea by using a CMG is introduced for balancing a heavy vehicle against continuous lateral acceleration. This assessment offers that CMG can stabilize vehicles with a constant rotational velocity of gyro. The sinusoidal gimbal motion generates a sinusoidal motion to maintain the balance the sprung mass in the upright position. with a stable period.

\section{System Set-Up}

In this paper, CMG consists of two gyroscopes moving in opposed directions for the opposed cornering speeds to each other to keep the vehicle in its upright position as shown in Figures 1 and 2. The precession of the gimbals is controlled by using a brake to fix the gimbal of flywheeel which is noneligible for the direction of cornering speed. To fix or set free the gimbal, the measurement of the direction of cornering is read by the on-off controller. Gyroscope generates the opposed-torque to balance the sprung mass motion in the upright position.

The precessions of the gyros occur $-\theta$ and $\theta$, respectively because the flywheels are rotating with the same angular speeds. For his reason, the equations of motion is based on two differential equations (10 and 11) of two degrees of 
freedom $\theta$, and $\varphi$, respectively. In Table 1, the vehicle's physical properties are given.

Table 1. Physical parameters of the vehicle

\begin{tabular}{|c|c|c|}
\hline Symbol & Numerical values & Description \\
\hline g & $9.80665 \mathrm{~m} / \mathrm{s}^{2}$ & Gravitational acceleration \\
\hline $\mathrm{R}$ & $21 \mathrm{~m}$ & the cornering radius of the vehicle \\
\hline $\mathrm{L}$ & $1.79 \mathrm{~m}$ & Mass centroid length of the sprung \\
\hline$m_{b}$ & 35565.951 kg & Sprung mass \\
\hline$I_{p}$ & $58.128 \mathrm{~kg} \cdot \mathrm{m}^{2}$ & The flywheel's rotary inertia \\
\hline $\mathrm{I}_{\mathrm{o}}$ & $30.822 \mathrm{~kg} \cdot \mathrm{m}^{2}$ & The flywheel's mass moment of inertia \\
\hline $\mathrm{I}_{\mathrm{gx}}$ & $20.255 \mathrm{~kg} \cdot \mathrm{m}^{2}$ & The gimbal's principal moment inertia \\
\hline $\mathrm{I}_{\mathrm{gy}}$ & $19.633 \mathrm{~kg} \cdot \mathrm{m}^{2}$ & The gimbal's principal moment inertia \\
\hline $\mathrm{I}_{\mathrm{gz}}$ & $2.461 \mathrm{~kg} \cdot \mathrm{m}^{2}$ & The gimbal's principal moments inertia \\
\hline $\mathrm{I}_{\mathrm{bx}}$ & $41954.056 \mathrm{~kg} \cdot \mathrm{m}^{2}$ & Principal moment inertia of the sprung \\
\hline$I_{\text {by }}$ & $308249.993 \mathrm{~kg} \cdot \mathrm{m}^{2}$ & Principal moment inertia of the sprung \\
\hline $\mathrm{I}_{\mathrm{bz}}$ & $315350.013 \mathrm{~kg} \cdot \mathrm{m}^{2}$ & Principal moment inertia of the sprung \\
\hline$\Omega$ & $-333.3 \mathrm{rad} / \mathrm{s}$ & Rotating speed of each flywheel \\
\hline$\dot{\alpha}$ & $0.05 \mathrm{rad} / \mathrm{s}$ & Cornering speed of vehicle \\
\hline $\mathrm{K}_{\varphi}$ & $250000 \mathrm{Nm} / \mathrm{rad}$ & Stiffness coefficient of torsional spring \\
\hline
\end{tabular}

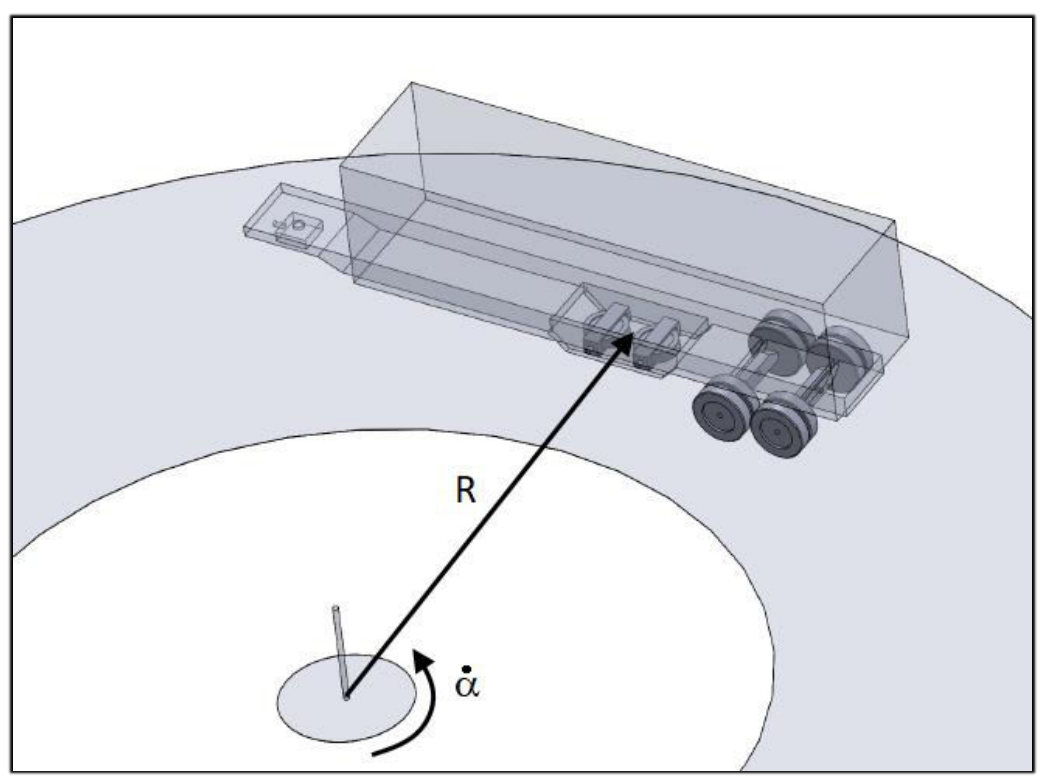

Figure 1. Physical model of a heavy vehicle. 

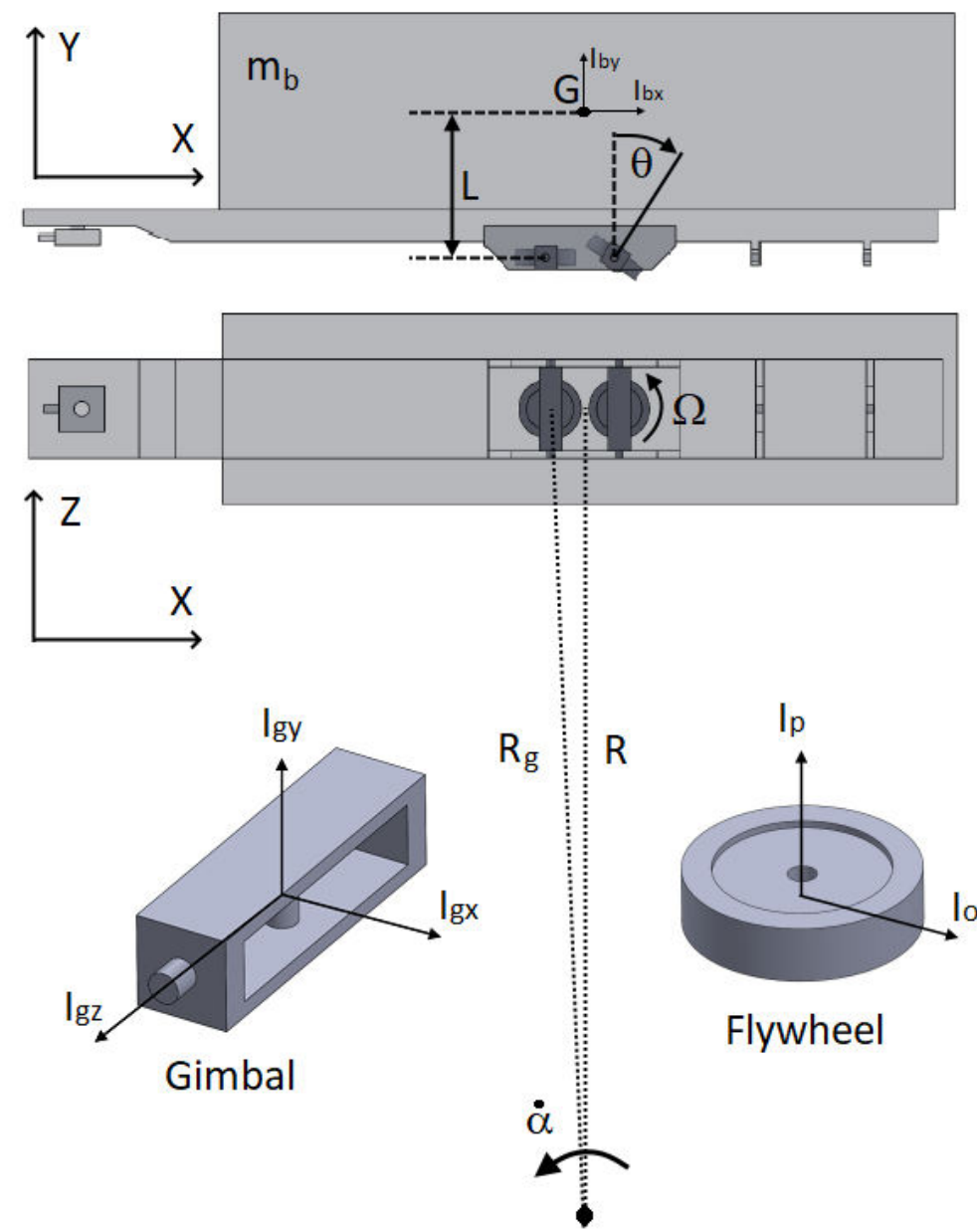

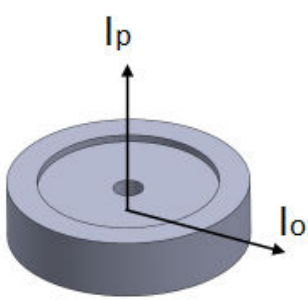

Flywheel

Figure 2. Gyroscopes in sprung mass, $m_{b}$ with length of the centroid $(G)$.

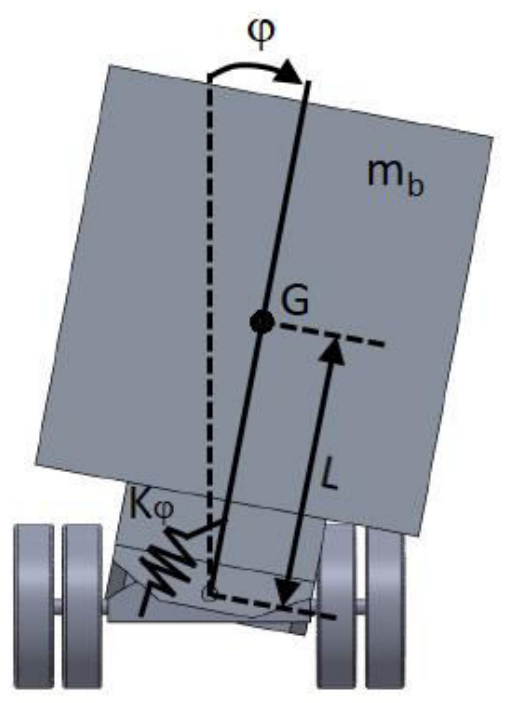

Figure 3. Vehicle rollover model in the vertical plane, joining a torsional spring. 


\section{Equations of Motion of Vehicle}

A simplified model of a heavy vehicle using two gyroscopes is seen in Figure 1.

The kinetic energies of the flywheels, gimbals, and the sprung are given as follows, respectively:

$T_{\text {flywheel_1 }}=\frac{1}{2} m_{d} \dot{\alpha}^{2} R_{g}{ }^{2}+\frac{1}{2} I_{o}\left[\dot{\theta}^{2}+(\dot{\varphi} \cos \theta+\dot{\alpha} \sin \theta)^{2}\right]+\frac{1}{2} I_{p}(\Omega-\dot{\varphi} \sin \theta+$ $\dot{\alpha} \cos \theta)^{2} ; \quad(1)$

$T_{\text {flywheel_2 }}=\frac{1}{2} m_{d} \dot{\alpha}^{2} R_{g}{ }^{2}+\frac{1}{2} I_{o} \dot{\varphi}^{2}+\frac{1}{2} I_{p}(-\Omega+\dot{\alpha})^{2} ;(2)$

$T_{\text {gimbal_ } 1}=\frac{1}{2} m_{g} \dot{\alpha}^{2} R_{g}{ }^{2}+\frac{1}{2} I_{g x}(\dot{\varphi} \cos \theta+\dot{\alpha} \sin \theta)^{2}+\frac{1}{2} I_{g y}(-\dot{\varphi} \sin \theta+$ $\dot{\alpha} \cos \theta)^{2}+\frac{1}{2} I_{g z} \dot{\theta}^{2}$

$T_{\text {gimbal_ } 2}=\frac{1}{2} m_{g} \dot{\alpha}^{2} R_{g}{ }^{2}+\frac{1}{2} I_{g x} \dot{\varphi}^{2}+\frac{1}{2} I_{g y} \dot{\alpha}^{2}$

$T_{\text {sprung mass }}=\frac{1}{2} m_{b}[(R+L \sin \varphi) \dot{\alpha}]^{2}+\frac{1}{2} m_{b}\left[(-L \dot{\varphi} \sin \varphi)^{2}+(L \dot{\varphi} \cos \varphi)^{2}\right]+$ $\frac{1}{2} I_{b x} \dot{\varphi}^{2}+\frac{1}{2} I_{b y} \dot{\alpha}^{2} \cos ^{2} \varphi+\frac{1}{2} I_{b z} \dot{\alpha}^{2} \sin ^{2} \varphi$

Herewith, the total kinetic energy can be given as: 
$T_{\text {total }}=T_{\text {flywheel_1 }}+T_{\text {flywheel_2 }}+T_{\text {gimbal_1 }}+T_{\text {gimbal_2 }}+T_{\text {sprung mass }} \cdot$

The mass center of gyroscopes is placed at the turning point of the sprung mass for a minimum potential energy. Thereby, the potential of the vehicle is

$V_{\text {total }}=m_{b} g L \cos \varphi+\frac{1}{2} K_{\varphi} \varphi^{2} .(7)$

Therefore, Lagrange's equations become

$\frac{d}{d t}\left(\frac{\partial T_{t o t a l}}{\partial \dot{\theta}}\right)-\frac{\partial T_{t o t a l}}{\partial \theta}+\frac{\partial V_{t o t a l}}{\partial \theta}=0$

$\frac{d}{d t}\left(\frac{\partial T_{\text {total }}}{\partial \dot{\varphi}}\right)-\frac{\partial T_{\text {total }}}{\partial \varphi}+\frac{\partial V_{\text {total }}}{\partial \varphi}=0$.

From above nonlinear Lagrange's equations of motion can be derived as follow:

$$
\begin{aligned}
& \left(I_{o}+I_{g z}\right) \ddot{\theta}-\left(I_{o}-I_{p}+I_{g x}-I_{g y}\right)(\dot{\varphi} \cos \theta+\dot{\alpha} \sin \theta)(-\dot{\varphi} \sin \theta+\dot{\alpha} \cos \theta)+ \\
& I_{p} \Omega(\dot{\varphi} \cos \theta+\dot{\alpha} \sin \theta)=0 ; \quad(10)
\end{aligned}
$$

$$
\begin{aligned}
& {\left[\left(I_{o}+I_{g x}\right) \cos ^{2} \theta+\left(I_{p}+I_{g y}\right) \sin ^{2} \theta+I_{o}+I_{g x}+m_{b} L^{2}+I_{b x}\right] \ddot{\varphi}+\left(I_{p}-I_{o}+\right.} \\
& \left.I_{g y}-I_{g x}\right)\left(2 \dot{\varphi} \dot{\theta} \cos \theta \sin \theta+\dot{\alpha} \dot{\theta} \sin ^{2} \theta-\dot{\alpha} \dot{\theta} \cos ^{2} \theta\right)-I_{p} \Omega \dot{\theta} \cos \theta-
\end{aligned}
$$


$m_{b} R L \dot{\alpha}^{2} \cos \varphi+\left(I_{b y}-I_{b z}-m_{b} L^{2}\right) \dot{\alpha}^{2} \cos \varphi \sin \varphi-m_{b} g L \sin \varphi+K_{\varphi} \varphi=0$.

Then, Equations (10) and (11) can be rearranged into the following forms for numeric solution;

$\ddot{\theta}=\frac{1}{\left(I_{o}+I_{g z}\right)}\left[\left(I_{o}-I_{p}+I_{g x}-I_{g y}\right)(\dot{\varphi} \cos \theta+\dot{\alpha} \sin \theta)(-\dot{\varphi} \sin \theta+\dot{\alpha} \cos \theta)-\right.$ $\left.I_{p} \Omega(\dot{\varphi} \cos \theta+\dot{\alpha} \sin \theta)\right] ;(12)$

$\ddot{\varphi}=$

$\frac{1}{\left(\left(I_{o}+I_{g x}\right) \cos ^{2} \theta+\left(I_{p}+I_{g y}\right) \sin ^{2} \theta+I_{o}+I_{g x}+m_{b} L^{2}+I_{b x}\right)}\left[\left(I_{o}-I_{p}+I_{g x}-\right.\right.$

$\left.I_{g y}\right)\left(2 \dot{\varphi} \dot{\theta} \cos \theta \sin \theta+\dot{\alpha} \dot{\theta} \sin ^{2} \theta-\dot{\alpha} \dot{\theta} \cos ^{2} \theta\right)+I_{p} \Omega \dot{\theta} \cos \theta+m_{b} R L \dot{\alpha}^{2} \cos \varphi-$ $\left.\left(I_{b y}-I_{b z}-m_{b} L^{2}\right) \dot{\alpha}^{2} \cos \varphi \sin \varphi+m_{b} g L \sin \varphi-K_{\varphi} \varphi\right]$. (13)

\subsection{Reduced Equations for Small Vibration of Sprung Mass about the Equilibrium Position}

Assume that a harmonic function is essential to stabilize the sprung mass against to a lateral acceleration due to the cornering speed. Thus, the roll of sprung mass, $\varphi(\mathrm{t})$ can be analyzed as a harmonic motion. Therefore, the motions may be selected as a frequency $\omega$ (Ünker, 2021): 
$\theta(t)=-\theta_{0} \sin (\omega t) ;(14)$

$\varphi(t)=\varphi_{0}[1-\cos (\omega t)]$

Through the Equation (15), the roll velocity is zero $(\dot{\varphi}=0)$ for the zero roll angle $(\varphi=0)$. Hence, the roll acceleration must have a small value $(\ddot{\varphi} \approx 0)$ with a low frequency according to the Equation (15). For the zero roll acceleration ( $\ddot{\varphi}=0$ ), ignoring the terms of a higher power for $\dot{\varphi} \approx 0$ near to the equilibrium position of sprung mass at the gimbal's zero precession $(\theta \approx 0$ and $\cos \theta=1)$, the Equations (10) and (11) can be reduced to;

$\left(I_{o}+I_{g z}\right) \ddot{\theta}-\left(I_{o}-I_{p}+I_{g x}-I_{g y}\right)(\dot{\varphi}+\dot{\alpha} \theta) \dot{\alpha}+I_{p} \Omega(\dot{\varphi}+\dot{\alpha} \theta)=0$

$$
\begin{aligned}
& \left(I_{p}-I_{o}+I_{g y}-I_{g x}\right)(-\dot{\alpha} \dot{\theta})-I_{p} \Omega \dot{\theta}-m_{b} R L \dot{\alpha}^{2} \cos \varphi+ \\
& \left(I_{b y}-I_{b z}-m_{b} L^{2}\right) \dot{\alpha}^{2} \cos \varphi \sin \varphi-m_{b} g L \sin \varphi+K_{\varphi} \varphi=0
\end{aligned}
$$

For a small amplitude of the roll vibrations $\left(\varphi_{0} \approx 0\right.$ and $\cos \varphi_{0}=1$ and $\left.\sin \varphi_{0}=\varphi_{0}\right)$ Equations (16) and (17) can be reduced into the following form; 


$$
\begin{aligned}
& \left(I_{o}+I_{g z}\right) \omega^{2} \theta_{0} \sin (\omega t)-\left(I_{o}-I_{p}+I_{g x}-I_{g y}\right)\left(\omega \varphi_{0}-\dot{\alpha} \theta_{0}\right) \dot{\alpha} \sin (\omega t)+ \\
& I_{p} \Omega\left(\omega \varphi_{0}-\dot{\alpha} \theta_{0}\right) \sin (\omega t)=0 ;(18)
\end{aligned}
$$

$$
\begin{aligned}
& \left(I_{p}-I_{o}+I_{g y}-I_{g x}\right) \dot{\alpha} \omega \theta_{0} \cos (\omega t)+I_{p} \Omega \omega \theta_{0} \cos (\omega t)-m_{b} R L \dot{\alpha}^{2}+\left(I_{b y}-\right. \\
& \left.I_{b z}-m_{b} L^{2}\right) \dot{\alpha}^{2} \varphi_{0}[1-\cos (\omega t)]-m_{b} g L \varphi_{0}[1-\cos (\omega t)]+K_{\varphi} \varphi_{0}[1- \\
& \cos (\omega t)]=0 .(19)
\end{aligned}
$$

Therefore, the gyroscope's precession amplitude, the sprung's roll amplitude, and the angular frequency can be derived from the above reduced equations as follows

$$
\theta_{0}=\frac{m_{b} R L \dot{\alpha}^{2}}{\left(I_{p}-I_{o}+I_{g y}-I_{g x}\right) \dot{\alpha} \omega+I_{p} \Omega \omega} ;
$$

$$
\varphi_{0}=\frac{m_{b} R L \dot{\alpha}^{2}}{\left(I_{b y}-I_{b z}-m_{b} L^{2}\right) \dot{\alpha}^{2}-m_{b} g L+K_{\varphi}} ;
$$

$$
\omega=\sqrt{\frac{\left(I_{o}-I_{p}+I_{g x}-I_{g y}\right) \dot{\alpha}^{2}-I_{p} \Omega \dot{\alpha}}{\frac{\left[\left(I_{p}-I_{o}+I_{g y}-I_{g x}\right) \dot{\alpha}+I_{p} \Omega\right]^{2}}{\left[-\left(I_{b y}-I_{b z}-m_{b} L^{2}\right) \dot{\alpha}^{2}+m_{b} g L-K_{\varphi}\right]}-\left(I_{o}+I_{g z}\right)}} .
$$


Equation (21) shows that roll amplitude is a constant for the constant cornering speed. Besides, optimum flywheel speed can be derived from Equation (22) as follow

$\Omega_{1,2}=\frac{-b \pm \sqrt{b^{2}-4 a c}}{2 a}$.

in which

$a=\frac{\omega^{2} I_{p}^{2}}{-\left(I_{b y}-I_{b z}-m_{b} L^{2}\right) \dot{\alpha}^{2}+m_{b} g L-K_{\varphi}} ;$

$b=I_{p} \dot{\alpha}+\frac{2 \omega^{2}\left(I_{p}-I_{o}+I_{g y}-I_{g x}\right) \dot{\alpha} I_{p}}{-\left(I_{b y}-I_{b z}-m_{b} L^{2}\right) \dot{\alpha}^{2}+m_{b} g L-K_{\varphi}} ;$

$c=\frac{\omega^{2}\left(I_{p}-I_{o}+I_{g y}-I_{g x}\right)^{2} \dot{\alpha}^{2}}{-\left(I_{b y}-I_{b z}-m_{b} L^{2}\right) \dot{\alpha}^{2}+m_{b} g L-K_{\varphi}}-\omega^{2}\left(I_{o}+I_{g z}\right)-\left(I_{o}-I_{p}+I_{g x}-I_{g y}\right) \dot{\alpha}^{2}$.

Hence, the optimum flywheel speed is obtained despite the nonlinear Lagrangian equations. A required amplitude of precession $\theta_{0}$ corresponding to a low velocity of flywheel can be derived for excisting cornering speeds as shown in Figure 4. 

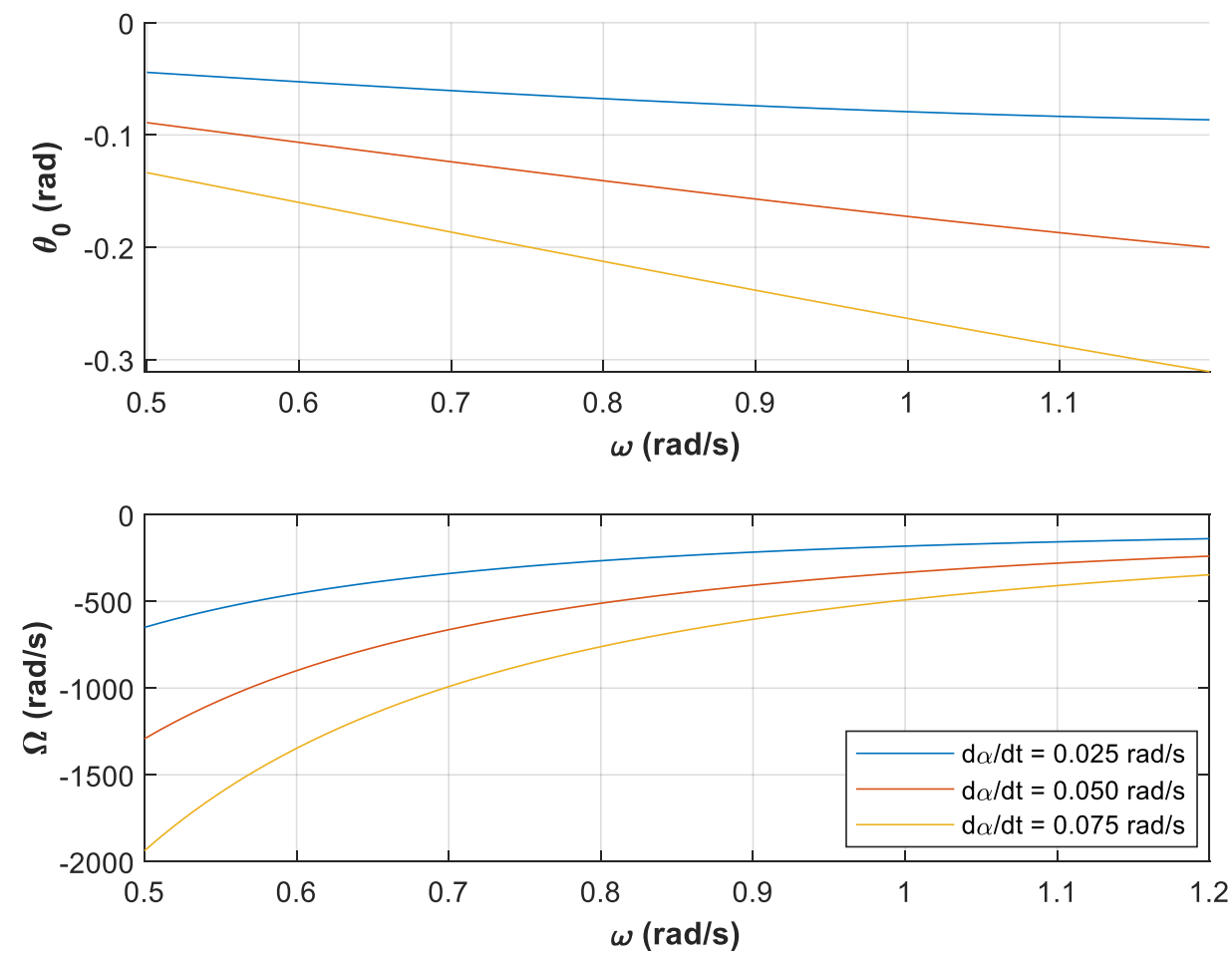

Figure 4. Effect of the frequency, $\omega$ on the gimbal amplitude, $\theta_{0}$ and the flywheel speed, $\Omega$ for different cornering speeds.

\section{Results}

In the following calculations, the Equations (12) and (13) can be solved by a numerical method (the fourth-order Runge-Kutta) using the physical properties given in Table 1. Optimum flywheel velocity can be selected through the equation (Eq. 23). In order to define the model given in Figures (1-3), With zero initial conditions, Lagrangian Equations were studied for the time step size of 0.001 second. Then, the theoretical simulations are corrected with RecurDyn results.

Figure 5 shows time responses for the varying flywheel velocities with the cornering speed of $\dot{\alpha}=0.05 \mathrm{rad} / \mathrm{s}$. The flywheel's speed was determined as constant for the linear rate of $1 \mathrm{rad} / \mathrm{s}$ and of time response amplitudes for 40 seconds was saved during each sweeping. As flywheel speed increases, the precession amplitude $\left(\theta_{0}\right)$ decreases, which indicates high control moment from 
the flywheel. Thus, the sprung mass roll $\left(\varphi_{0}\right)$ decreases and runs into robust motion after a required flywheel speed.
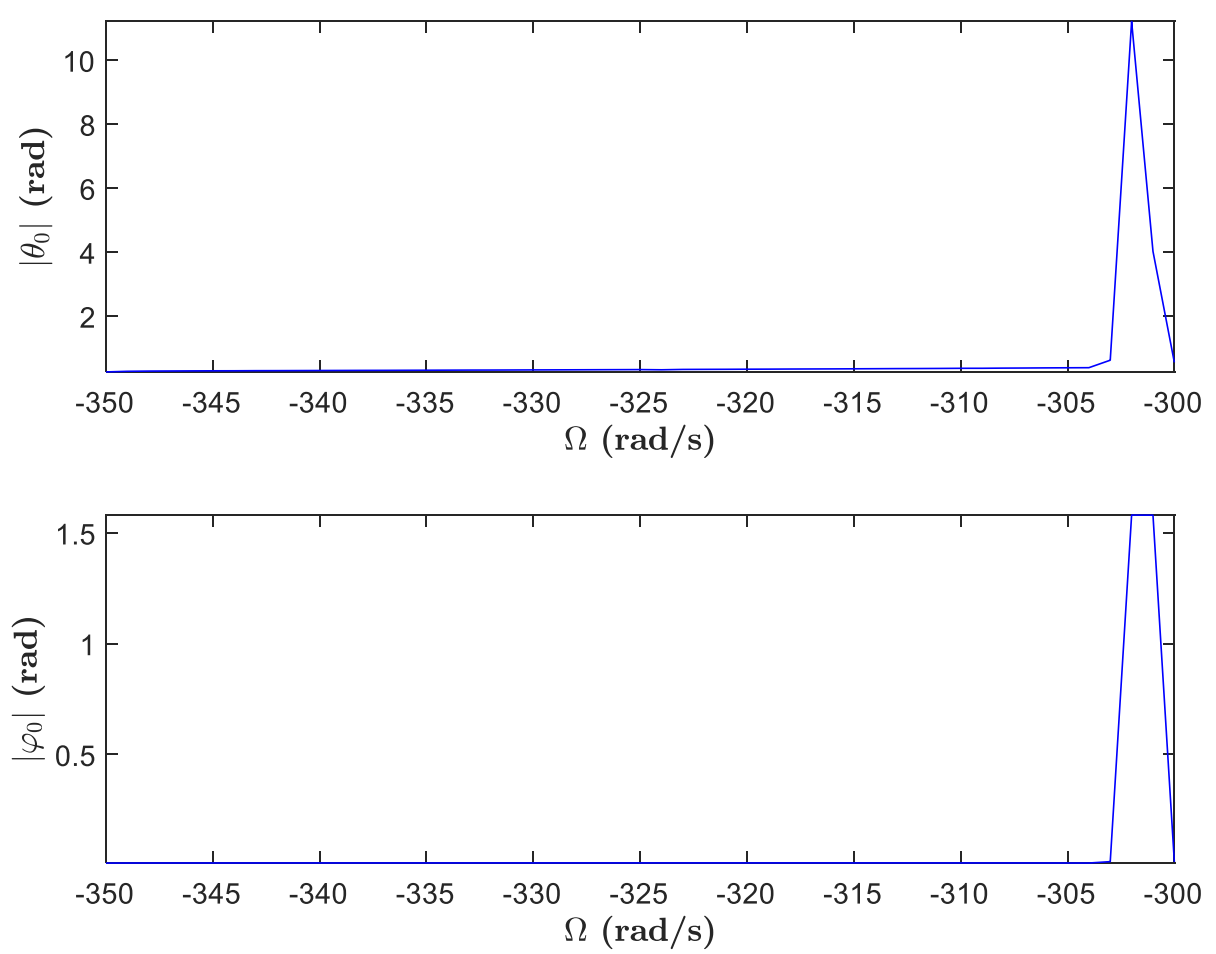

Figure 5. The flywheel speed effect, $\Omega$ for $\dot{\alpha}=0.05 \mathrm{rad} / \mathrm{s}$

Figure 6 shows the cornering speed curves at $\Omega=-333 \mathrm{rad} / \mathrm{s}$.Tthe roll amplitude $\varphi_{0}$ and precession of gimbal increase and run into unstable motion while increasing the cornering speed. However, the roll mitigation performance is improved using a recuired flywheel speed for a certain cornering speed . 

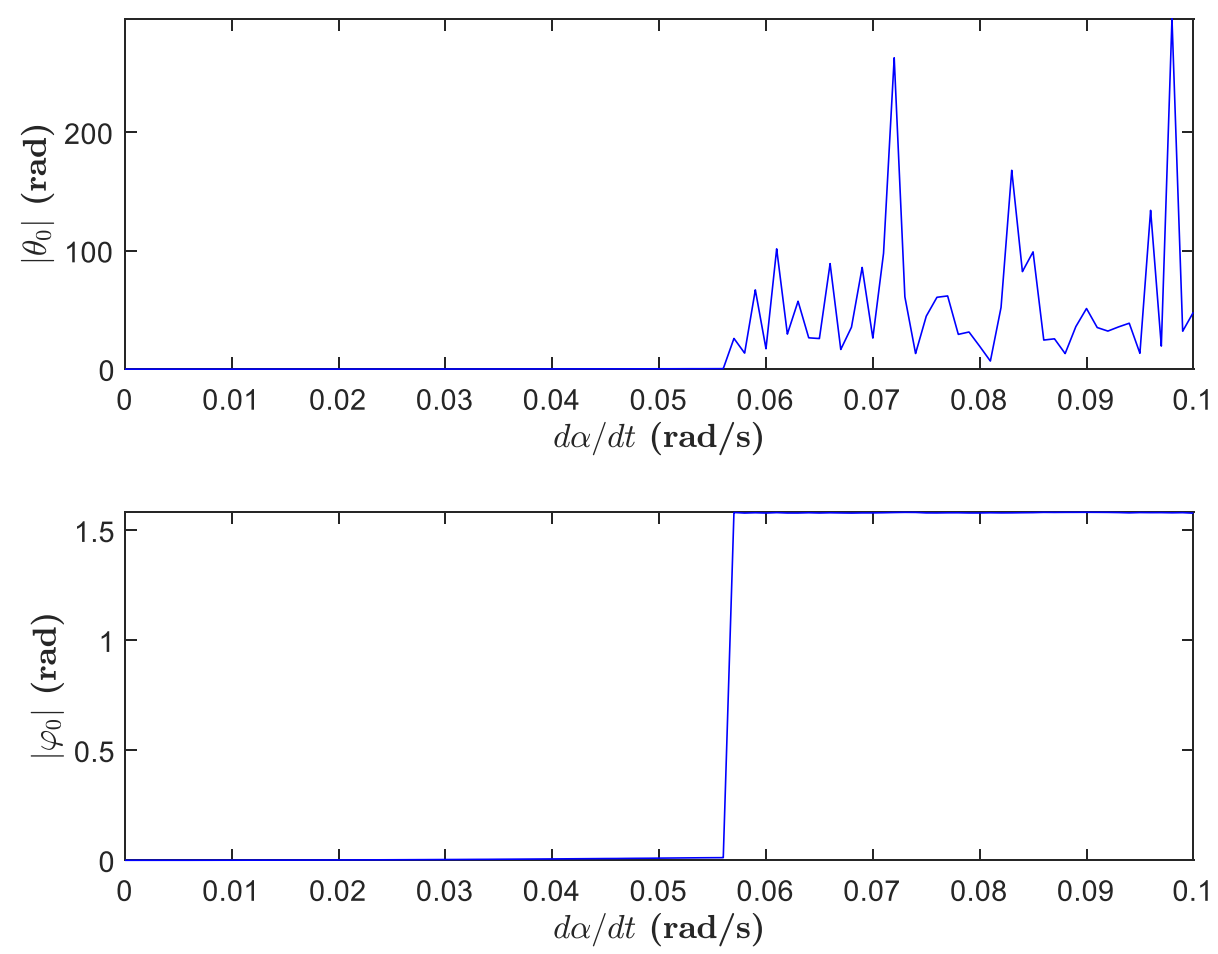

Figure 6. Effect of $\dot{\alpha}$ for $\Omega=-333.3 \mathrm{rad} / \mathrm{s}$.

Lagrange's equations' the stationary $(\Omega=0 \mathrm{rad} / \mathrm{s})$ and controlled $(\Omega=-333.3 \mathrm{rad} / \mathrm{s})$ CMG displacements are shown in Figure 7. The flywheel speed $\Omega=-333.3 \mathrm{rad} / \mathrm{s}$ for the frequency $\omega=1 \mathrm{rad} / \mathrm{s}$ show that $C M G$ keeps the body stable. However, the stationary $\mathrm{CMG}(\Omega=0 \mathrm{rad} / \mathrm{s})$ loses control. So, the $\varphi$-roll response of the sprung can be balanced under the continuous lateral acceleration with a constant flywheel speed. 

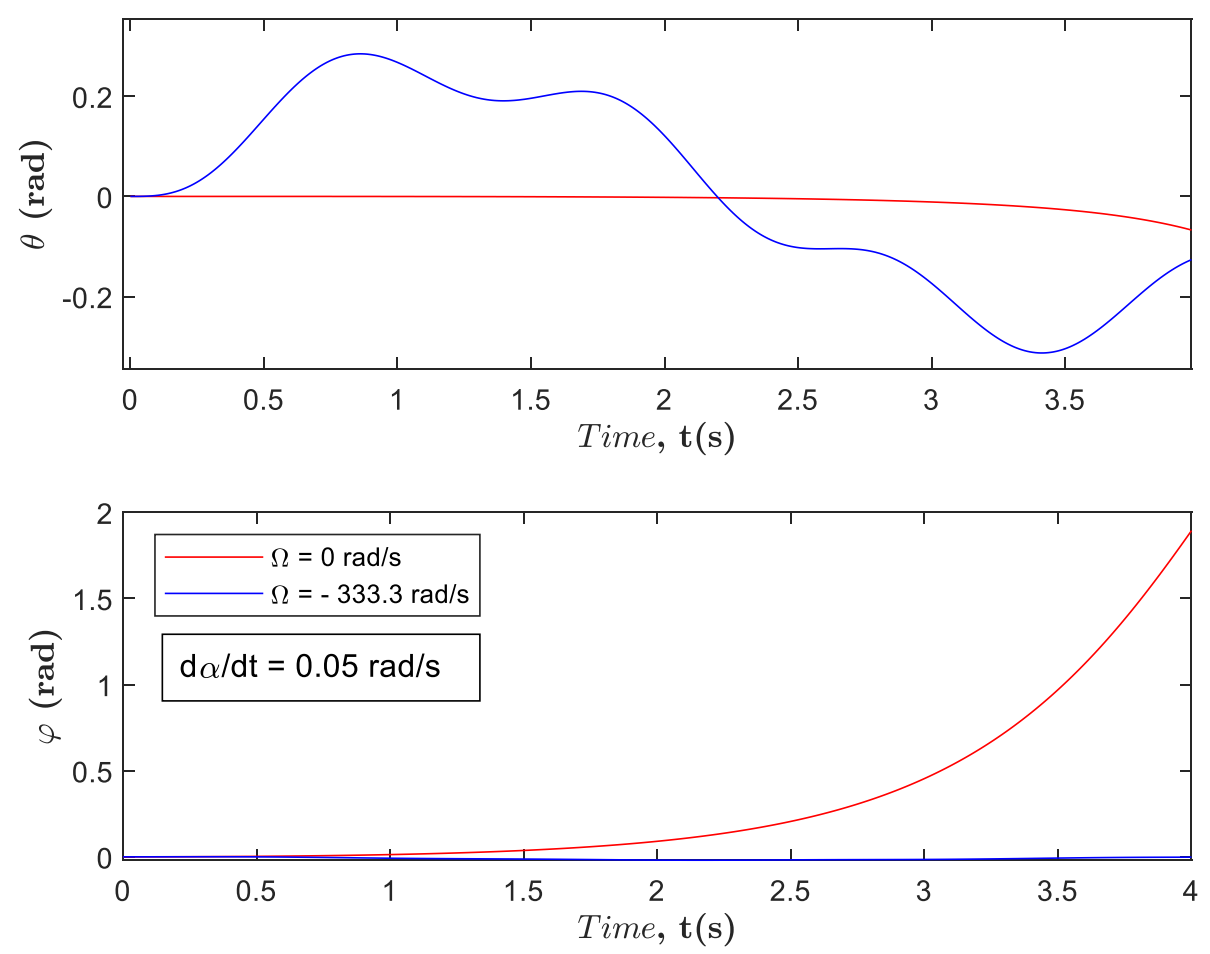

Figure 7. Time response curves of the flywheel speeds, $\Omega=0$ and $-333.3 \mathrm{rad} / \mathrm{s}$ with $\dot{\alpha}=0.05$ $\mathrm{rad} / \mathrm{s}$ for the frequency $\omega=1 \mathrm{rad} / \mathrm{s}$.

In Figures 8, 9 and 10, Lagrange's data were compared with the RecurDyn simulations for different flywheel speeds with the same cornering speed $\dot{\alpha}=0.05$ $\mathrm{rad} / \mathrm{s}$. The comparisons of sprung mass and gyroscope are studied that the theoretical results and RecurDyn simulations are almost the same and the inclinations of the simulations agreed well with Figure 4 derived from the Equations (20-24). Besides, similar simulations can be also achived using the Equations (14) and (15). Since the precession amplitude of gimbal $\theta_{0}$ increases, the flywheel needs low angular speed. Nevertheless, the precession amplitude of gyro $\theta_{0}$ should have a small value for a steady motion as possible. 

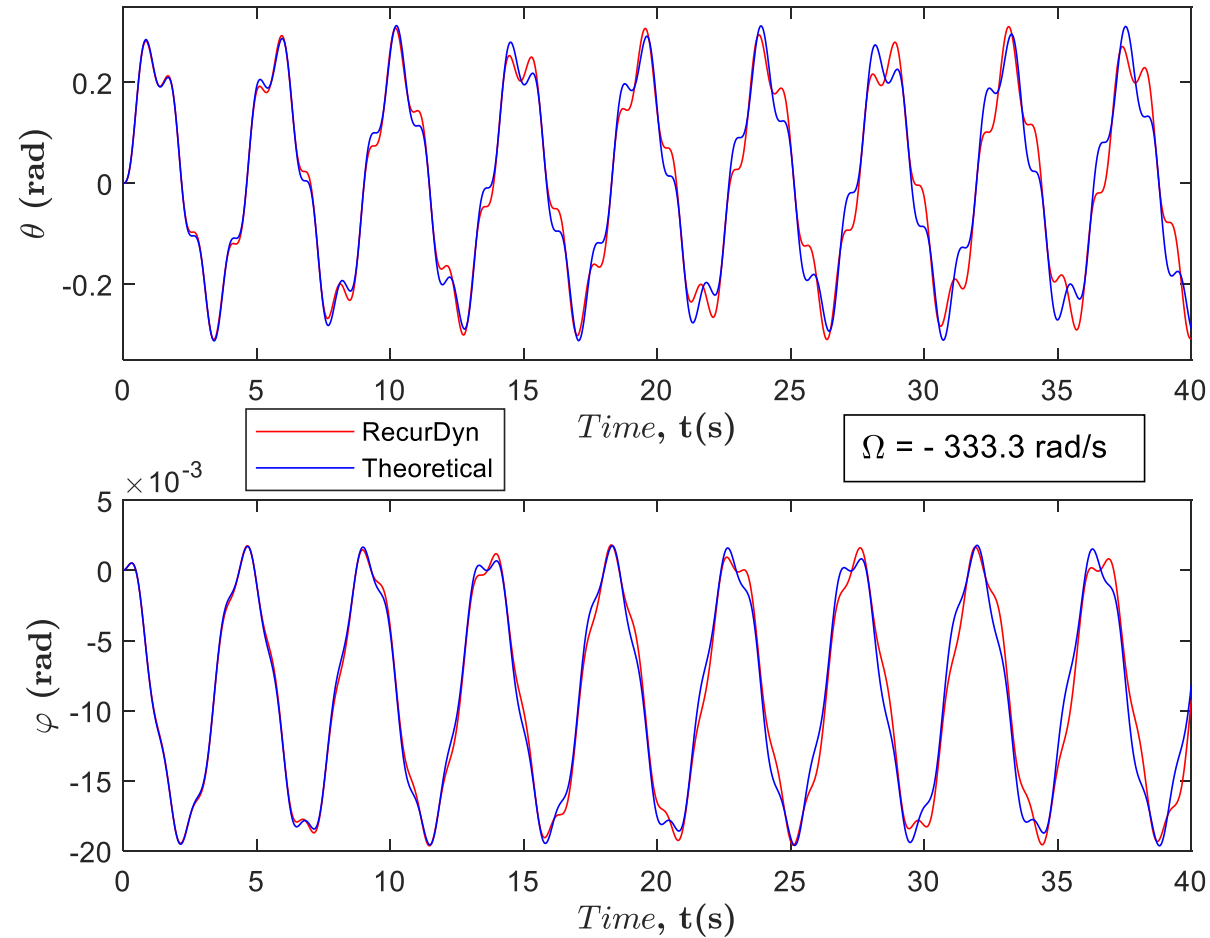

Figure 8. Comparisons of RecurDyn and the theoretical simulations for $\dot{\alpha}=0.05 \mathrm{rad} / \mathrm{s}$ with the frequency $\omega=1 \mathrm{rad} / \mathrm{s}$.
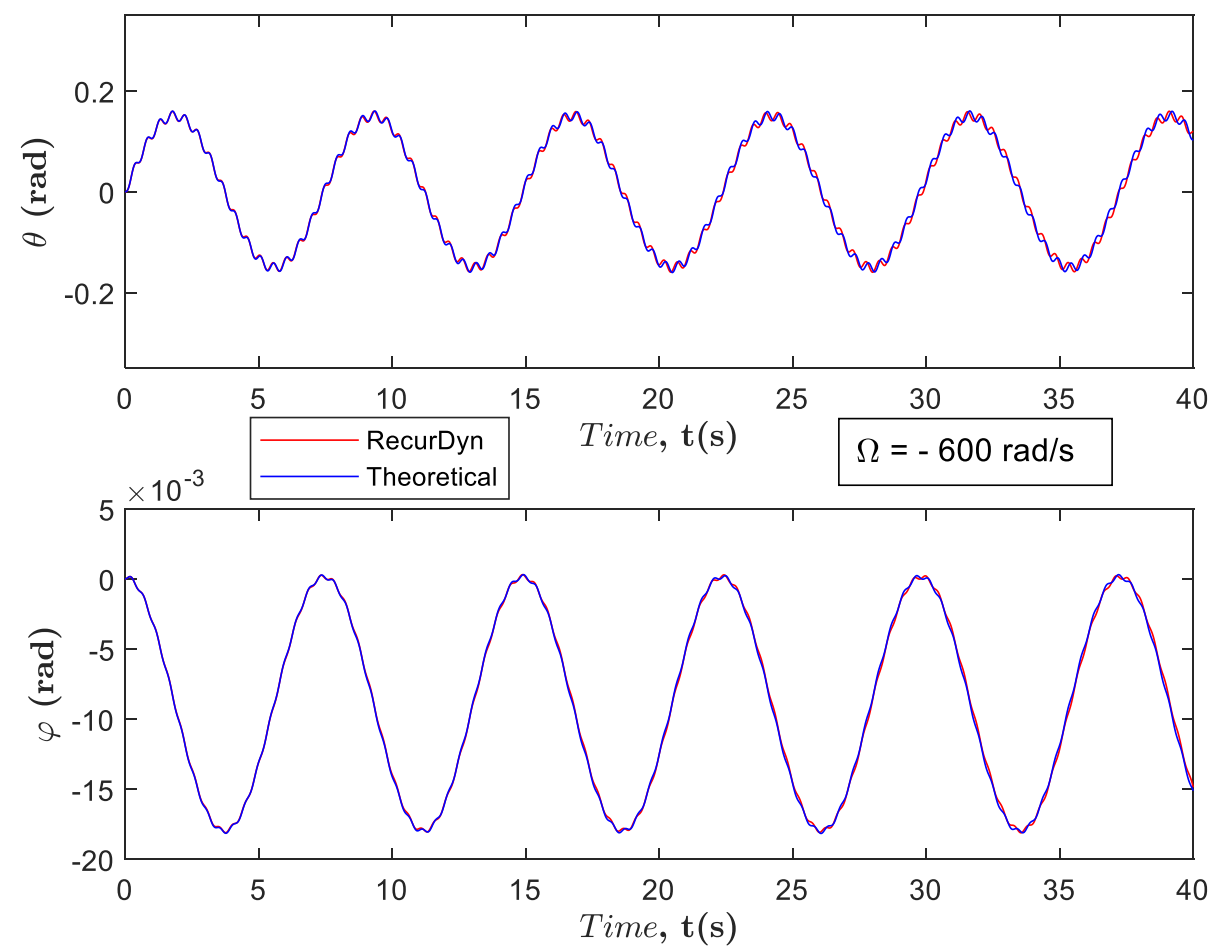

Figure 9. Comparisons of RecurDyn and the theoretical simulations for the frequency for $\dot{\alpha}=$ $0.05 \mathrm{rad} / \mathrm{s}$ with the frequency $\omega=0.74 \mathrm{rad} / \mathrm{s}$. 

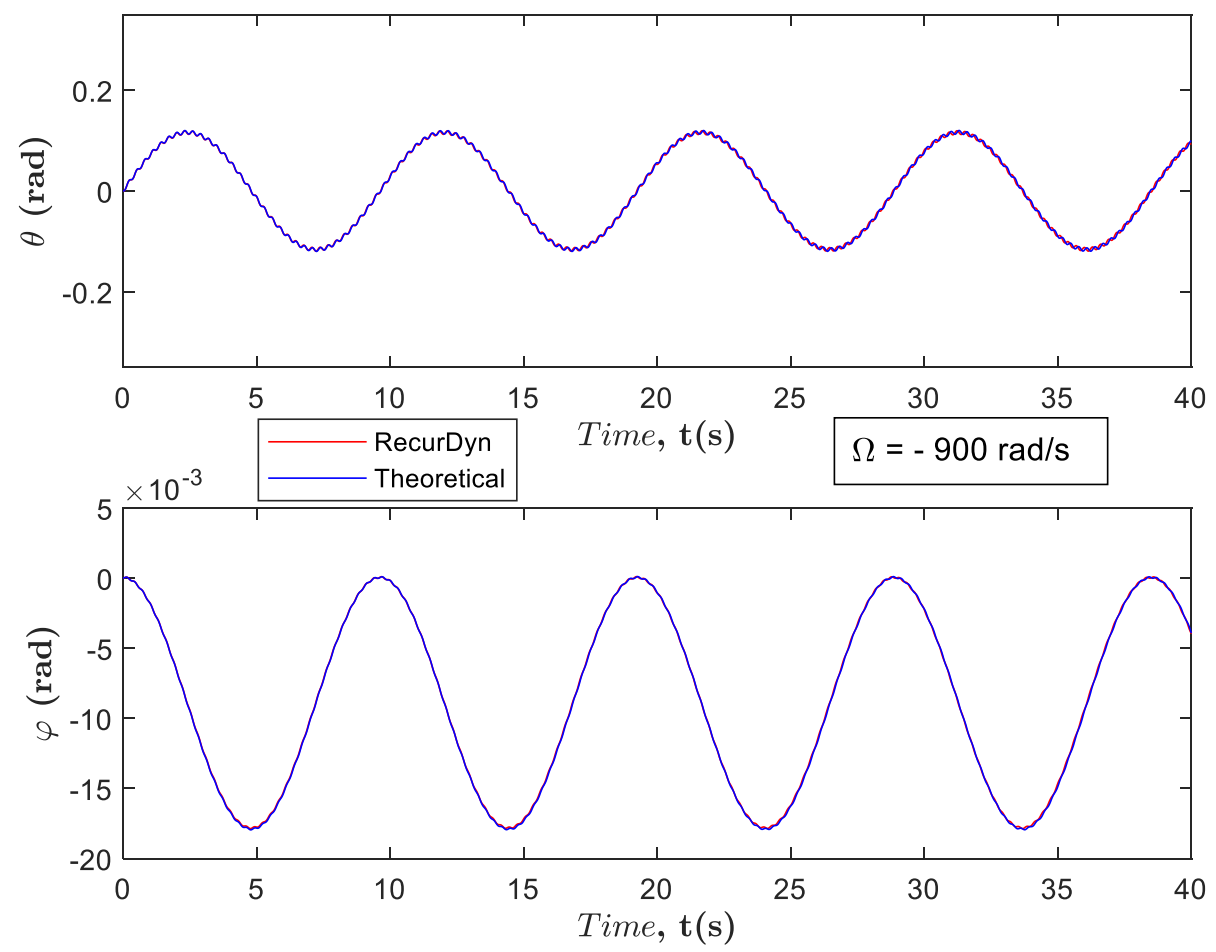

Figure 10. Comparisons of RecurDyn and the theoretical simulations for $\dot{\alpha}=0.05 \mathrm{rad} / \mathrm{s}$ with the frequency $\omega=0.6 \mathrm{rad} / \mathrm{s}$.

\section{Conclusion}

With the help of a CMG, a heavy vehicle is aimed for driving fast under the lateral forces due to the cornering speed. The problem of maintaining constant moments against to inertia forces of cornering speed was solved with an optimum flywheel speed of gyro. The constant speed of flywheel can stabilize the sprung's motion in the vertical position. The optimum speeds of gyros have been derived from Lagrangian equations.

The results show that the vehicle can corner without losing sprung's stability through a CMG. The improvement clearly depends on the angular momentum of the flywheel. The precession of the gimbals must be controlled by an on-off controller using a measured direction of cornering speed of vehicle to fix or set 
free the adequate gimbal. Noneligible gimbal for the direction of cornering speed must be fixed during cornering.

The flywheel requires low angular velocity since the amplitude of gimbal precession increases. However, the precession amplitude of gimbal should have small value as possible for a stable harmonic motion under the continuous lateral acceleration.

Besides, the theoretical results is verified by RecurDyn, indicates that the RecurDyn simulations agree well with Laglangian ones. Furthermore, the comparisons are stable and almost the same.

\section{Declaration of conflicting interests}

The author declared no potential conflicts of interest with respect to the research, authorship, and/or publication of this article.

\section{Funding}

The author received no financial support for the research, authorship, and/or publication of this article.

\section{REFERENCES}

Chen B. and Peng H, (2001) Differential-braking-based rollover prevention for sports utility vehicles with human-in-the-loop evaluations. Vehicle System Dynamics 36, (4-5): 359-389.

Hac A (2002) Influence of active chassis systems on vehicle propensity to maneuver-induced rollovers. SAE Paper 2002-01-0967. 
Irdayanti Y, Kusumanto RD, Anisah M, et al. (2020) Ultrasonic Sensor Application As A Performance Enhancement of Robot Two Wheels. J. Phys.: Conf. Ser. 1500 (012007).

Larimi SR, Zarafshan P, and Moosavian SAA (2015) A New Stabilization Algorithm for a Two-Wheeled Mobile Robot Aided by Reaction Wheel. Journal of Dynamic Systems, Measurement, and Control 137(1): 011009 (8 pages)

Lee AY (2002) Coordinated control of steering and anti-roll bars to alter vehicle rollover tendencies. ASME Journal of Dynamic Systems, Measurement, and Control 124: 127-132.

Lin SC, Tsai CC and Huang HC (2011) Adaptive robust selfbalancing and steering of a two-wheeled human transportation vehicle. J Intell Robot Syst 62(1): 103-123.

Mirzaeinejad H and Mirzaei M (2014) Optimization of nonlinear control strategy for anti-lock braking system with improvement of vehicle directional stability on split- $\mu$ roads, Trans. Research Part C 46: 1-15.

Ruan X and Chen J (2010) H1 robust control of self-balancing two wheeled robot." In: 8th World congress on intelligent control and automation, Jinan, China, 7-9 July 2010, pp.6524-6527. IEEE. 
Takahashi J, Yamakado M and Saito S (2013) Evaluation of preview Gvectoring control to decelerate a vehicle prior to entry into a curve, Int. J. of Automotive Technology 14 (6): 921-926.

Tchamna R, Youn E and Youn I (2014) Combined control effects of brake and active suspension control on the global safety of a full-car nonlinear model, Vehicle System Dynamics 52 (1) : 69-91.

Ungoren AY and Peng H, (2004 ) Evaluation of vehicle dynamic control for rollover prevention. International Journal of Automotive Technology 5(2): $115-122$.

Ünker F (2020) Tuned gyro pendulum stabilizer for control of vibrations in structures. International Journal of Acoustics and Vibration 25(3): $355-362$.

Ünker F (2021) Proportional control moment gyroscope for two-wheeled self-balancing robot. Journal of Vibration and Control 0(0): 1-9.

Xu J, Shang S, Qi H, et al. (2016) Simulative investigation on head injuries of electric self-balancing scooter riders subject to ground impact. Accid Anal Prev 89:128-141.

Yoon J, Cho W, Koo B and Yi K (2009) Unified chassis control for rollover prevention and lateral stability. IEEE Trans. on Vehicular Technology 58( 2): 596-609. 